\title{
CATETER CENTRAL DE INSERÇÃO PERIFÉRICA: REVISÃO SISTEMÁTICA
}

\author{
PERIPHERALLY INSERTED CENTRAL CATHETER: SYSTEMATIC REVIEW
}

\author{
Cassiane Martins $^{\mathrm{a}^{*}}$, Gleidson Brandão Oselame ${ }^{\mathrm{b} *}$, Eduardo Borba Neves ${ }^{\mathrm{c} *}$ \\ akssi.m@hotmail.com, bgoselame@ics.curitiba.pr.gov.br, cborbaneves@hotmail.com \\ *Centro Universitário Campos de Andrade - Curitiba (PR), Brasil \\ **Universidade Tecnológica Federal do Paraná (UTFPR) - Curitiba (PR), Brasil
}

Data de recebimento do artigo: 07/05/2015

Data de aceite do artigo: 02/10/2015

\section{RESUMO}

Introduçáo: O Peripherally Inserted Central Venous Catheter (PICC) está entre os avanços tecnológicos e terapêuticos em expansão na saúde. Ele apresenta a possibilidade de estabelecer terapia endovenosa de prolongada duração, com redução do número de punçóes e inserção na beira do leito por enfermeiro habilitado. Objetivo: Descrever o perfil predominante do paciente com uso do PICC, principais diagnósticos e especialidades médicas que fazem uso do cateter, condutas associadas, tempo médio de permanência e locais de inserção. Método: Revisão sistemática em banco de dados eletrônicos da Literatura Latino-Americana e do Caribe em Ciências de Saúde, Scientific Eletronic Library Online, Base de Dados da Enfermagem e por meio do buscador Google Acadêmico. Resultados e Conclusóes: Identificou-se a prevalência de indivíduos do sexo masculino, prematuros, baixo peso e com menos de 7 dias de vida no momento da inserção do PICC. As indicações foram administração de nutrição parenteral total, antibioticoterapia e medicaçóes. Como complicaçóes, o rompimento, obstrução, infecção, prematuridade e afecçóes respiratórias. A média de permanência do PICC foi 11,3 dias. De 19 estudos, 16 eram em neonatologia. As veias mais acessadas foram a basílica e cefálica.

Palavras-chave: Enfermagem; Unidades de Terapia Intensiva; cateteres; obstrução do cateter.

\section{ABSTRACT}

Introduction: Peripherally Inserted Central Venous Catheter (PICC) is among the expanding technological and therapeutic advances in health. It presents the possibility of establishing intravenous therapy of prolonged duration, reducing the number of punctures and insertion at bedside by qualified nurses. Objective: To describe the predominant profile of patients using PICC, the main diagnoses and medical specialties that make use of the catheter, associated procedures, average duration of stay, and insertion sites. Method: Systematic review in the electronic databases of the Latin American and Caribbean Health Sciences Literature, Scientific Electronic Library Online, Database of Nursing, and through Google Scholar search engine. Results and Conclusions: We identified a prevalence of premature, underweight males less than 7 days old on the date of insertion of PICC. The main indications for the device were administering total parenteral nutrition, antibiotics, and medication. The complications were disruption, obstruction, infection, prematurity, and respiratory conditions. The average duration of stay with the catheter was 11.3 days. Out of 19 studies, 16 were in neonatology. The most accessed veins were the basilica and cephalic.

Keywords: Nursing; Intensive Care Units; catheters; obstruction of the catheter. 


\section{Introdução}

Nos hospitais, as Unidades de Terapias Intensivas (UTI) são destinadas a tratar pacientes com risco de morte, seja por doenças ou outras condiçôes clínicas que resultem em instabilidade ou disfunçáo do(s) sistema(s) fisiológico $(s)^{1}$. As UTI neonatais têm como finalidade o cuidado individualizado visando a qualidade de vida, crescimento e desenvolvimento saudáveis de recém-nascidos $(\mathrm{RN})$ com quadros clínicos de alta complexidade, submetidos a procedimentos invasivos, com base no cuidado neonatal que exige da equipe conhecimento e aperfeiçoamento constante ${ }^{2}$.

O perfil das crianças internadas em UTI neonatais tem sido modificado graças ao avanço tecnológico aliado ao crescente desenvolvimento tecnocientífico que, juntos, propiciaram uma demanda de cuidados mais complexos, com a utilização de procedimentos invasivos e aprimoramento dos cuidados prestados, garantindo, dessa forma, a sobrevivência de $\mathrm{RN}$ de alto risco ${ }^{3}$. Especialmente neonatos prematuros (idade gestacional (IG) inferior a 37 semanas) e/ou com baixo peso (peso ao nascimento inferior a $2.500 \mathrm{~g})^{4-5}$.

A terapêutica intravenosa, através de um acesso venoso seguro e funcional, insere-se nesse contexto como um recurso primordial na assistência desses recém-natos, que geralmente necessitam de medicamentos por um tempo prolongado 6 . O Peripherally Inserted Central Venous Catheter (PICC) está entre esses avanços tecnológicos e terapêuticos em expansão dentro da UTI. Ele apresenta inúmeros benefícios, entre eles, a possibilidade de estabelecer terapia endovenosa de prolongada duração, com redução do número de punções e inserção na beira do leito pelo enfermeiro habilitado através de qualificação e capacitação profissional, gerando um cuidado legitimado e conferindo autonomia profissional ${ }^{7}$.

Nesse sentido, o PICC constitui-se a primeira escolha para acesso venoso central após o cateterismo umbilical, sobressaindo-se ao convencional cateter venoso central (CVC). Dessa forma, o artigo $1^{\circ}$ da Resolução $n^{\circ}$ 258/2001, do Conselho Federal de Enfermagem, define a competência técnica e legal para o enfermeiro inserir o PICC e o artigo $2^{\circ}$ da mesma resolução diz que é responsabilidade do enfermeiro após qualificação e/ ou capacitação profissional o desempenho da atividade ${ }^{8}$.

Destaca-se que a porcentagem de complicaçóes associadas ao uso do PICC é considerável, variando de $15 \%$ a $48 \%$, representada por eventos como oclusão, extravasamento, migração da ponta do cateter e trombose, essas intercorrências resultam na redução do tempo de permanência do cateter e consequente insucesso na implementação terapêutica medicamentosa e sobrevida dos $\mathrm{RN}^{9}$.
Portanto, esse estudo teve como objetivo descrever o perfil predominante do paciente com uso do PICC, principais diagnósticos e especialidades médicas que fazem uso do cateter, condutas associadas, tempo médio de permanência e locais de inserção.

\section{Metodologia}

Tratou-se de uma revisão sistemática que consiste em uma importante ferramenta da prática baseada em evidências, permite atualização dos profissionais, promove a melhoria da qualidade da assistência de enfermagem ao cliente e auxilia na tomada de decisóes referentes à assistência à saúde ${ }^{10}$.

Foram contemplados neste estudo artigos publicados e indexados em banco de dados eletrônicos da Literatura Latino-Americana e do Caribe em Ciências de Saúde (LILACS), do Scientific Eletronic Library Online (ScIELO), da Base de Dados da Enfermagem (BDENF) e demais estudos que não constam nessas bases de dados por meio do buscador Google Acadêmico. Os artigos foram buscados através dos descritores: "enfermagem", "Unidades de Terapia Intensiva", "infecçôes relacionadas a cateter", "cateteres", "obstruçâo do cateter", conforme os Descritores em Ciências da Saúde (DeCS, 2014). A pergunta norteadora do estudo foi: quais as variáveis influentes no sucesso do procedimento de inserção do PICC?

A pesquisa foi delimitada para artigos publicados na base de dados supracitados, e atendeu aos seguintes critérios de inclusão: publicação entre o período de 2004 a 2014, em periódicos nacionais e internacionais, disponíveis no idioma português e que abordavam a temática PICC.

A coleta de dados foi realizada em agosto de 2014 . Os artigos inicialmente receberam uma leitura exploratória, que tem como finalidade identificar a validade do documento para a pesquisa. Posteriormente, foi realizada a leitura seletiva a fim de determinar se o material apresentava importância para a pesquisa, e a seguir, procedeu-se a leitura analítica do material selecionado de forma a ordenar e sumarizar as informaçóes sobre o objeto de estudo e, por fim, realizou-se a leitura interpretativa para relacionar as afirmaçóes dos autores com o problema proposto.

A análise dos dados provenientes da coleta foi agrupada por temas, de acordo com a abordagem dos autores, sendo discutida com a literatura atual sobre o assunto. Foram extraídas as seguintes variáveis dos estudos que compuseram a base dessa revisão: perfil predominante do paciente com uso do PICC, principais diagnósticos e especialidades médicas que fazem uso do cateter e condutas associadas, tempo médio de permanência e locais de inserção. 


\section{Resultados}

Foram selecionados, inicialmente, 397 estudos. Após aplicação dos critérios de exclusão e da leitura exploratória e seletiva, mostraram-se pertinentes 36 deles. Destes, 19 artigos tiveram seus principais resultados diretamente relacionados ao objetivo do estudo em questáo, apresentando maior número de variáveis referentes à caracterização dos pacientes submetidos ao PICC, principais diagnósticos, especialidades médicas, complicaçóes decorrentes do uso do PICC e local de inserção do cateter, de forma que tais dados foram elencados no Quadro 1.

Dos dezenove estudos, dezesseis tinham a neonatologia como especialidade médica, dois tratavam de adultos (em UTI e ambiente hospitalar) e um era na área de oncologia e hematologia. As complicaçôes mais citadas foram rompimento, obstrução e infecção. As veias mais acessadas foram as veias basílica e cefálica. O Quadro 2 explicita essas variáveis.

Quadro 1: Caracterização dos pacientes submetidos ao PICC conforme os estudos analisados.

\begin{tabular}{|c|c|c|c|c|c|c|c|}
\hline \multirow{2}{*}{ Estudo } & \multicolumn{2}{|c|}{ Sexo } & \multirow{2}{*}{$\begin{array}{c}\text { Idade } \\
\text { Gestacional }\end{array}$} & \multirow{2}{*}{$\begin{array}{l}\text { Idade na } \\
\text { inserçáo }\end{array}$} & \multirow{2}{*}{ Peso } & \multirow{2}{*}{ Indicação } & \multirow{2}{*}{$\begin{array}{c}\text { Tempo de } \\
\text { permanência } \\
\text { do PICC }\end{array}$} \\
\hline & M & $\mathbf{F}$ & & & & & \\
\hline $\begin{array}{l}\text { BERGAMI et al. } \\
(2012) \text {. }\end{array}$ & $57,5 \%$ & $42,5 \%$ & - & $\begin{array}{c}10 \text { a } 14 \text { anos } \\
(33,8 \%) ; 5 \text { a } 9 \\
\text { anos }(25,6 \%) ; 1 \\
\text { a } 4 \text { anos }(19,4 \%)\end{array}$ & - & $\begin{array}{c}\text { QT } \\
\text { Glicose } \\
\text { Medicamento } \\
\text { Sangue } \\
\text { NP }\end{array}$ & $\begin{array}{c}\text { Média = 49,9 } \\
\text { dias. Mediana } \\
=26 \text { dias } \\
\text { Variação = } \\
1-398 \text { dias }\end{array}$ \\
\hline ROSA et al. (2014) & - & - & - & $\begin{array}{c}<\text { de } 7 \text { dias } \\
(69,56 \%)\end{array}$ & $\begin{array}{c}<2.500 \mathrm{~g} \\
(75,35 \%) ; \\
1.501 \text { a } 2.000 \mathrm{~g} \\
(30,43 \%)\end{array}$ & $\begin{array}{c}\text { ATB }(40,57 \%) \\
\text { Soro }(24,63 \%) \mathrm{NP} \\
(23,18 \%)\end{array}$ & $\begin{array}{c}\text { Média }=7 \text { dias } \\
\text { Variação }= \\
\text { 3-28 dias }\end{array}$ \\
\hline $\begin{array}{l}\text { COSTA et al. } \\
\quad(2012)\end{array}$ & $65,5 \%$ & $34,5 \%$ & $\begin{array}{c}<37 \text { semanas } \\
(95,2 \%)\end{array}$ & $\begin{array}{c}5,3 \text { dias } \pm \\
12,91(\mathrm{DP})\end{array}$ & $\begin{array}{c}\text { Média= } \\
1.777,3 \mathrm{~g} \pm \\
643(\mathrm{DP})\end{array}$ & $\begin{array}{l}\text { ATB }(78,6 \%) \\
\operatorname{NP}(57,1 \%)\end{array}$ & $\begin{array}{c}\text { Média }=11,8 \\
\text { dias } \pm 12,8 \\
(\mathrm{DP})\end{array}$ \\
\hline $\begin{array}{l}\text { CABRAL et al. } \\
\quad(2013)\end{array}$ & $58,0 \%$ & $42,0 \%$ & $\begin{array}{l}<35 \text { semanas } \\
(87 \%), 25 \text { a } 30 \\
\text { semanas }(55 \%)\end{array}$ & $\begin{array}{c}1 \text { a } 5 \text { dias } \\
(85 \%)\end{array}$ & $\begin{array}{c}<2.300 \mathrm{~g} \\
(94 \%) ; 1.101 \\
\text { a } 1.700 \mathrm{~g} \\
(47 \%)\end{array}$ & $\begin{array}{c}\text { NP e } \\
\text { ATB }(43 \%) \\
\text { TE }(26 \%)\end{array}$ & $\begin{array}{c}6 \text { a } 10 \text { dias } \\
(35 \%) ; 10 \\
\text { a } 15 \text { dias } \\
(35 \%) ; 16 \text { a } \\
20 \text { dias }(13 \%)\end{array}$ \\
\hline $\begin{array}{l}\text { COSTA et al. } \\
\quad(2013)\end{array}$ & $59,0 \%$ & $41,0 \%$ & $\begin{array}{c}\text { Média }=34 \\
\text { semanas } \pm 4,1 \\
(D P)\end{array}$ & $\begin{array}{c}\text { Média }=10,3 \\
\text { dias } \pm 18,1 \\
(\mathrm{DP})\end{array}$ & $\begin{array}{c}\text { Média }=1.900 \\
\mathrm{~g} \pm 849,3 \mathrm{~g} \\
(\mathrm{DP})\end{array}$ & $\begin{array}{l}\text { Prematuros } \\
\text { ATB }\end{array}$ & - \\
\hline $\begin{array}{l}\text { BAGGIO; BAZZI; } \\
\text { BILIBIO (2010) }\end{array}$ & $60,0 \%$ & $40,0 \%$ & - & $\begin{array}{c}0 \text { a } 28 \text { dias } \\
(71,6 \%), 29 \\
\text { dias a } 15 \text { anos } \\
(28,4 \%)\end{array}$ & $\begin{array}{l}\text { De } 652 \text { g ( } 28 \\
\text { semanas) a } 70 \\
\mathrm{~kg}(15 \text { anos })\end{array}$ & $\begin{array}{c}\text { NP } \\
\text { Medicamento }\end{array}$ & $\begin{array}{l}\text { Média }=14,5 \\
\text { dias } \\
\text { Variação }=1 \mathrm{a} \\
72 \text { dias }\end{array}$ \\
\hline $\begin{array}{l}\text { FREITAS; NUNES } \\
\qquad(2009)\end{array}$ & $42,8 \%$ & $57,2 \%$ & $\begin{array}{c}30 \text { a } 34 \text { sema- } \\
\text { nas }(57,2 \%)\end{array}$ & $\begin{array}{l}\text { Logo após o } \\
\text { nascimento }\end{array}$ & $\begin{array}{c}<2.200 \mathrm{~g} \\
(64,3 \%)\end{array}$ & $\begin{array}{c}\text { NP } \\
\text { ATB } \\
\text { Medicamento }\end{array}$ & $\begin{array}{c}\text { Média }=10,5 \\
\text { dias } \\
\text { Variação }=3 \mathrm{a} \\
17 \text { dias }\end{array}$ \\
\hline $\begin{array}{c}\text { COSTA et al. } \\
(2010)\end{array}$ & $42,9 \%$ & $57,1 \%$ & $\begin{array}{c}<35 \text { semanas } \\
(82 \%)\end{array}$ & $\begin{array}{l}1 \text { a } 7 \text { dias } \\
(67,8 \%)\end{array}$ & $\begin{array}{l}<1.500 \mathrm{~g} \\
(71,4 \%)\end{array}$ & - & - \\
\hline $\begin{array}{l}\text { GOMES et al. } \\
\quad(2010)\end{array}$ & $21,4 \%$ & $78,6 \%$ & - & - & - & $\begin{array}{c}\text { NP } \\
\text { Medicamento } \\
(78,6 \%)\end{array}$ & $\begin{array}{c}\text { Média }=15 \\
\text { dias } \\
\text { Mediana }=14 \\
\text { dias }\end{array}$ \\
\hline $\begin{array}{l}\text { MOTTA et al. } \\
\quad(2011)\end{array}$ & - & - & - & - & $\begin{array}{l}\text { De } 500 \mathrm{~g} \\
\text { a } 3.600 \mathrm{~g} . \\
\text { Maioria < } \\
2.500 \mathrm{~g}\end{array}$ & $\begin{array}{c}\text { NP } \\
\text { ATB } \\
\text { Medicamento }\end{array}$ & $\begin{array}{c}10 \text { dias } \\
\text { ou menos } \\
(60,2 \%) ; \\
11 \text { a } 20 \text { dias } \\
(31,5 \%)\end{array}$ \\
\hline
\end{tabular}

continua... 
Quadro 1: Continuação.

\begin{tabular}{|c|c|c|c|c|c|c|c|}
\hline \multirow{2}{*}{ Estudo } & \multicolumn{2}{|c|}{ Sexo } & \multirow{2}{*}{$\begin{array}{c}\text { Idade } \\
\text { Gestacional }\end{array}$} & \multirow{2}{*}{$\begin{array}{l}\text { Idade na } \\
\text { inserçáo }\end{array}$} & \multirow{2}{*}{ Peso } & \multirow{2}{*}{ Indicaçáo } & \multirow{2}{*}{$\begin{array}{c}\text { Tempo de } \\
\text { permanência } \\
\text { do PICC }\end{array}$} \\
\hline & $\mathbf{M}$ & $\mathbf{F}$ & & & & & \\
\hline $\begin{array}{l}\text { LAMBLET et al. } \\
(2005)\end{array}$ & $57,5 \%$ & $42,5 \%$ & - & $\begin{array}{c}\text { Média }=60,3 \\
\text { anos. De } 70 \text { a } \\
79 \text { anos }(25 \%)\end{array}$ & - & $\begin{array}{l}\text { ATB }(65 \%) \\
\text { Acesso difícil } \\
\quad(25 \%)\end{array}$ & $\begin{array}{l}\text { Pacientes de } \\
20 \text { a } 59 \text { anos } \\
\text { Média de } \\
20,1 \text { dias }\end{array}$ \\
\hline $\begin{array}{l}\text { CAMARGO et al. } \\
(2008)\end{array}$ & $59,4 \%$ & $40,6 \%$ & $\begin{array}{c}\text { Média }=31,6 \\
\text { semanas. } \leq 32 \\
\text { semanas } \\
(62,2 \%)\end{array}$ & $\begin{array}{c}\text { Média }=4,3 \\
\text { dias; } 1 \text { a } 3 \text { dias } \\
(48,7 \%) ; 4 \text { a } 7 \\
\text { dias }(37,8 \%)\end{array}$ & $\begin{array}{c}\text { Média }=1.289 \mathrm{~g} ; \\
\quad \leq 1.500 \mathrm{~g} \\
(72,3 \%)\end{array}$ & $\begin{array}{c}\mathrm{NP}(94,6 \%) \\
\text { ProstaglandinaE1 } \\
(5,4 \%)\end{array}$ & - \\
\hline COSTA et al. (2013) & $59,1 \%$ & $40,9 \%$ & $\begin{array}{c}\text { Média }=34,1 \\
\text { semanas, } \\
\text { DP 4,09 }\end{array}$ & $\begin{array}{l}\text { Média = 10,6 } \\
\text { dias, DP 18,7 }\end{array}$ & $\begin{array}{c}\text { Média }= \\
1.091,5 \mathrm{~g}, \mathrm{DP} \\
860,7\end{array}$ & - & - \\
\hline $\begin{array}{l}\text { DUARTE et al. } \\
(2013)\end{array}$ & $53,4 \%$ & $46,6 \%$ & $\begin{array}{c}\leq 35 \text { semanas } \\
69,7 \%\end{array}$ & $\begin{array}{c}0 \text { a } 7 \text { dias } \\
(68,4 \%) ; 31 \text { a } 61 \\
\operatorname{dias}(15,5 \%)\end{array}$ & $\begin{array}{c}\leq 1.500 \mathrm{~g} \\
(42,8 \%)\end{array}$ & $\begin{array}{l}\operatorname{ATB}(72,9 \%) \\
\operatorname{NP}(31,3 \%)\end{array}$ & $\begin{array}{c}8 \text { a } 14 \text { dias } \\
(34,4 \%) . \\
\text { Tempo máximo } \\
=68 \text { dias }\end{array}$ \\
\hline REIS et al. (2011) & - & - & - & - & - & $\mathrm{TE}$ & $\begin{array}{c}1 \text { a } 2 \text { semanas. } \\
\text { Média }=7,7\end{array}$ \\
\hline $\begin{array}{l}\text { MONTES et al. } \\
\text { (2011) }\end{array}$ & - & - & $\begin{array}{c}\text { Média = 31,9 } \\
\pm 4,1 \text { semanas } \\
\text { (DP) Variação = } \\
35 \text { a } 40 \text { semanas }\end{array}$ & - & $\begin{array}{c}\text { Média }=1.639,5 \\
\pm 632,7 \mathrm{~g} . \mathrm{De} \\
715 \mathrm{~g} \text { a } 2.826 \mathrm{~g}\end{array}$ & - & $\begin{array}{c}\text { Média }=8,8 \\
\text { dias } \pm 6,1 \text { dias } \\
(\mathrm{DP}) ; \text { de } 6 \text { a } 10 \\
\text { dias }(38,2 \%)\end{array}$ \\
\hline $\begin{array}{l}\text { BAIOCCO; SILVA } \\
(2010)\end{array}$ & $70,7 \%$ & $29,3 \%$ & - & $\begin{array}{c}\text { Média }=61,5 \\
\text { anos } \pm 17,8 \\
(\mathrm{DP}) ; \text { de } 19 \text { a } 93 \\
\text { anos }\end{array}$ & - & $\begin{array}{c}\text { ATB }(54,1 \%), \\
\text { QT }(20,1 \%)\end{array}$ & $\begin{array}{c}\text { Mediana = } 13 \\
\text { dias; de } 7 \text { a } 24 \\
\text { dias }(50 \%)\end{array}$ \\
\hline $\begin{array}{l}\text { DÓREA et al. } \\
(2011)\end{array}$ & $73,3 \%$ & $26,7 \%$ & $\begin{array}{c}<30 \text { semanas } \\
(37,8 \%)\end{array}$ & $\leq 3 \operatorname{dias}(53,3 \%)$ & $\begin{array}{c}<2.000 \mathrm{~g} \\
(62,2 \%)\end{array}$ & $\begin{array}{c}\text { ATB e } \\
\operatorname{NP}(55,5 \%)\end{array}$ & $\begin{array}{l}\text { Média = 9,42 } \\
\text { dias. Variação } \\
\text { de } 1 \text { a } 41 \text { dias }\end{array}$ \\
\hline $\begin{array}{l}\text { FRANCESCHI; } \\
\text { CUNHA (2010) }\end{array}$ & $53,9 \%$ & $46,1 \%$ & $\begin{array}{c}\text { Média }=33,6 \pm \\
4,6(\mathrm{DP})\end{array}$ & Mediana $=1 \mathrm{dia}$ & $\begin{array}{c}1.993,4 \pm 943,6 \\
\text { (DP) }\end{array}$ & - & - \\
\hline
\end{tabular}

Legenda: $\mathrm{DP}=$ Desvio Padrão, $\mathrm{NP}=$ Nutrição Parenteral, $\mathrm{QT}=$ Quimioterapia, $\mathrm{ATB}=$ antibioticoterapia, $\mathrm{TE}$ = terapia endovenosa.

Quadro 2: Principais diagnósticos, especialidades médicas, complicações decorrentes do uso do PICC e local de inserção.

\begin{tabular}{|c|c|c|c|c|}
\hline Estudo & Principais diagnósticos & $\begin{array}{l}\text { Especialidade } \\
\text { Médica }\end{array}$ & Principais Complicaçóes & $\begin{array}{l}\text { Local de Inserçáo } \\
\text { (principais veias) }\end{array}$ \\
\hline $\begin{array}{l}\text { BERGAMI et al. } \\
(2012)\end{array}$ & $\begin{array}{l}\text { Leucemia Linfoide Aguda } \\
(36,9 \%) \text {; Leucemia } \\
\text { Mieloide Aguda }(13,8 \%) ; \\
\text { Tumor Ósseo }(6,3 \%)\end{array}$ & $\begin{array}{l}\text { Oncologia e } \\
\text { Hematologia }\end{array}$ & $\begin{array}{c}\text { Infecção }(50,8 \%) \text {; Oclusão } \\
\text { (30,3\%); Infiltração }(10,7 \%) \text {; } \\
\text { Tração do cateter }(4,9 \%) ; \\
\text { Trombose }(3,3 \%)\end{array}$ & $\begin{array}{l}\text { V. Basílica }(50 \%) ; \text { V. } \\
\text { cefálica }(20 \%) ; \text { V. Cubital } \\
\text { mediana }(19,4 \%)\end{array}$ \\
\hline ROSA et al. (2014) & $\begin{array}{l}\text { Prematuridade }(44,92 \%) \text {; } \\
\text { Asfixia }(30,43 \%) \text {; Distresse } \\
\text { Respiratório }(13,04 \%)\end{array}$ & Neonatologia & $\begin{array}{c}\text { Rompimento (7,24\%); } \\
\text { Obstrução (5,79\%); Infecção } \\
(4,34 \%) \text {; Infiltração }(2,89 \%)\end{array}$ & $\begin{array}{l}\text { V. Cefálica }(27,53 \%) ; \mathrm{V} \text {. } \\
\text { Basílica mediana }(24,63 \%) ; \\
\text { V. Safena }(15,94 \%)\end{array}$ \\
\hline COSTA et al. (2012) & $\begin{array}{c}\text { Afecçóes Respiratórias } \\
(69 \%) ; \text { Choque/Sepse } \\
(14,2 \%) ; \text { Cardiopatias } \\
(7,1 \%) .\end{array}$ & Neonatologia & $\begin{array}{c}\text { Obstrução (13,1\%); Ruptura } \\
\text { (9,5\%); Edema (7,1\%); } \\
\text { Suspeita Infecção }(6 \%)\end{array}$ & - \\
\hline $\begin{array}{c}\text { CABRAL et al. } \\
\text { (2013) }\end{array}$ & Prematuridade (46\%) & Neonatologia & $\begin{array}{l}\text { Perda do acesso }(25 \%) \\
\text { Sinais Flogísticos }(21 \%)\end{array}$ & $\begin{array}{l}\text { MMSS (88\%), Basílica, } \\
\text { Mediana cubital, radial e } \\
\text { cefálica. }\end{array}$ \\
\hline
\end{tabular}

continua... 
Quadro 2: Continuação.

\begin{tabular}{|c|c|c|c|c|}
\hline Estudo & Principais diagnósticos & $\begin{array}{l}\text { Especialidade } \\
\text { Médica }\end{array}$ & Principais Complicaçôes & $\begin{array}{l}\text { Local de Inserçáo } \\
\text { (principais veias) }\end{array}$ \\
\hline COSTA et al. (2013) & $\begin{array}{l}\text { Prematuridade }(79,1 \%) ; \\
\text { Desconforto Respiratório } \\
(67,6 \%) \text {; Gemelaridade } \\
(23,4 \%) \text {; Sepse }(22,1 \%)\end{array}$ & Neonatologia & & $\begin{array}{c}\text { V. Axilar (32,5\%); V. } \\
\text { Basílica }(18,9 \%) ; \text { V. } \\
\text { Cefálica }(14,2 \%) ; \text { V. Safena } \\
(9,4 \%)\end{array}$ \\
\hline $\begin{array}{l}\text { BAGGIO; BAZZI; } \\
\text { BILIBIO (2010) }\end{array}$ & $\begin{array}{l}\text { Prematuridade }(60,8 \%) ; \\
\text { Cardiopatia congênita/ } \\
\text { cirurgia cardíaca }(12,8 \%) ; \\
\text { Insuficiência Respiratória } \\
\text { (10,4\%); Cirurgia } \\
\text { Pediátrica }(5,6 \%)\end{array}$ & $\begin{array}{l}\text { Neonatologia e } \\
\text { Pediatria }\end{array}$ & $\begin{array}{c}\text { Obstrução }(25 \%) \text {; Infiltração } \\
(18 \%) ; \text { Suspeita conta- } \\
\text { minação (16,6\%); Tração } \\
(13,9 \%) ; \text { Ruptura }(11,2 \%) ; \\
\text { Flebite }(4,2 \%)\end{array}$ & $\begin{array}{l}\text { V. Basílica }(23,8 \%) ; \text { V. } \\
\text { Cefálica }(19,4 \%) ; \text { V. } \\
\text { Auricular }(18,2 \%) ; \text { V. } \\
\text { Jugular Ext. }(13,6 \%)\end{array}$ \\
\hline $\begin{array}{l}\text { FREITAS; NUNES } \\
\qquad(2009)\end{array}$ & - & Neonatologia & $\begin{array}{c}\text { Obstrução (14,3\%); Infecção } \\
(14,3 \%)\end{array}$ & $\begin{array}{c}\text { V. Basílica }(71,6 \%) ; \text { V. } \\
\text { Axilar (14,2\%); V. Safena } \\
\text { (7,1\%) }\end{array}$ \\
\hline COSTA et al. (2010) & $\begin{array}{l}\text { Prematuridade (displasia } \\
\text { pulmonar, retinopatia, } \\
\text { restrição crescimento) }\end{array}$ & Neonatologia & - & - \\
\hline GOMES et al. (2010) & $\begin{array}{c}\text { Gastrosquise }(28,6 \%) ; \\
\text { Prematuridade }(28,6 \%) \text {; } \\
\text { Onfalocele }(7,1 \%)\end{array}$ & $\begin{array}{l}\text { Neonatologia cirúrgi- } \\
\text { ca e não cirúrgica }\end{array}$ & $\begin{array}{c}\text { Obstrução e Ruptura } \\
(21,4 \%) \text {; Complicação infec- } \\
\text { ciosa }(14,3 \%)\end{array}$ & $\begin{array}{c}\text { V. Basílica }(42,9 \%) \text {; V. } \\
\text { Jugular }(14,3 \%), \text { V. Safena } \\
(14,3 \%)\end{array}$ \\
\hline MOTTA et al. (2011) & - & Neonatologia & $\begin{array}{l}\text { Rompimento do cateter } \\
(23 \%) \text {; Extravasamento } \\
(23 \%) \text {; Obstruçáo }(18 \%)\end{array}$ & $\begin{array}{c}\text { V. Basílica }(24,7 \%) \text {; V. } \\
\text { Cefálica }(21,9 \%) ; \text { V. Safena } \\
(12,3 \%)\end{array}$ \\
\hline $\begin{array}{l}\text { LAMBLET et al. } \\
\qquad(2005)\end{array}$ & $\begin{array}{l}\text { AVE (15\%); Pneumonia } \\
(15 \%) ; \text { Neoplasia }(10 \%) ; \\
\text { ICC }(10 \%) ; \text { DPOC }(7,5 \%)\end{array}$ & UTI Adulto & $\begin{array}{l}\text { Exteriorização }(7,5 \%) ; \\
\text { Flebite }(5 \%) ; \text { Obstrução } \\
\qquad(2,5 \%)\end{array}$ & $\begin{array}{c}\text { V. Basílica }(65 \%) ; \mathrm{V} \text {. } \\
\text { Cefálica }(25 \%) ; \mathrm{V} . \text { Cubital } \\
\text { média }(10 \%)\end{array}$ \\
\hline $\begin{array}{l}\text { CAMARGO et al. } \\
\qquad(2008)\end{array}$ & $\begin{array}{c}\text { SDR }(56,8 \%) \text {; Síndrome do } \\
\text { Pulmão Úmido (29,7\%); } \\
\text { Cardiopatia Congênita } \\
(5,4 \%)\end{array}$ & Neonatologia & - & - \\
\hline COSTA et al. (2013) & - & Neonatologia & - & - \\
\hline $\begin{array}{l}\text { DUARTE et al. } \\
\qquad(2013)\end{array}$ & $\begin{array}{c}\text { Sepse }(72,5 \%) ; \\
\text { Prematuridade }(70,5 \%) ; \\
\text { Baixo Peso }(69,8 \%) ; \text { SDR }^{*} \\
(16,5 \%)\end{array}$ & Neonatologia & Infecção $(15,8 \%)$ & $\begin{aligned} & \text { MMSS* }^{*}(83,1 \%) ; \text { MMII* } \\
&(8,6 \%) ; \text { Região Cervical } \\
&(4,1 \%)\end{aligned}$ \\
\hline REIS et al. (2011) & $\begin{array}{l}\text { DRP* }(31,9 \%) ; \text { Asfixia } \\
(9,9 \%) \text {; Cirurgia }(9,9 \%)\end{array}$ & Neonatologia & $\begin{array}{l}\text { Migração (18\%); Rotura } \\
(7,1 \%) ; \text { Infecçáo }(6,3 \%)\end{array}$ & - \\
\hline $\begin{array}{l}\text { MONTES et al. } \\
\text { (2011) }\end{array}$ & $\begin{array}{c}\text { Alteraçóes: Respiratórias } \\
\text { (61\%); Infecciosas }(17,1 \%) ; \\
\text { Metabólicas }(9,8 \%)\end{array}$ & Neonatologia & $\begin{array}{c}\text { Obstrução }(30,9 \%) \text {; Ruptura } \\
\begin{array}{c}(16,4 \%) ; \text { Exteriorizaçáo } \\
(9,1 \%) ; \text { Extravasamento } \\
(5,5 \%)\end{array}\end{array}$ & $\begin{array}{l}\text { V. Safena }(27,3 \%) ; \mathrm{V} \text {. } \\
\text { Cefálica }(23,6 \%) ; \mathrm{V} \text {. } \\
\text { Basílica }(18,2 \%)\end{array}$ \\
\hline $\begin{array}{l}\text { BAIOCCO; SILVA } \\
(2010)\end{array}$ & $\begin{array}{c}\text { Anemia, aplasia da medula, } \\
\text { leucemia, HIV e linfoma } \\
(17,9 \%) \text {; CA de reto, } \\
\text { fístulas infecção, obstruçáo } \\
\text { e suboclusão intestinal, } \\
\text { pancreatite e peritonite } \\
(13,1 \%)\end{array}$ & $\begin{array}{l}\text { Adultos em ambiente } \\
\text { hospitalar }\end{array}$ & $\begin{array}{c}\text { Migração }(28,8 \%) \text {; Febre } \\
(20,3 \%) ; \text { Obstrução } \\
(15,3 \%) \text {; Tracionamento } \\
(10,2 \%) \text {; Dobra, pseudofle- } \\
\text { bite, resistência, arrancamen- } \\
\text { to }(6,8 \%)\end{array}$ & $\begin{array}{c}\text { Veia basílica }(62,9 \%) \text { e Veia } \\
\text { Cefálica }(37,1 \%)\end{array}$ \\
\hline
\end{tabular}

continua.. 
Quadro 2: Continuação.

\begin{tabular}{|c|c|c|c|c|}
\hline Estudo & Principais diagnósticos & $\begin{array}{c}\text { Especialidade } \\
\text { Médica }\end{array}$ & Principais Complicaçóes & $\begin{array}{l}\text { Local de Inserçáo } \\
\text { (principais veias) }\end{array}$ \\
\hline DÓREA et al. (2011) & $\begin{array}{c}\text { Afecção Respiratória } \\
(75,5 \%) ; \text { Gastrointestinal } \\
(20 \%)\end{array}$ & Neonatologia & $\begin{array}{c}\text { Ruptura }(15,4 \%) \text {; Oclusão } \\
\text { (11\%); Remoção Acidental } \\
(4,4 \%)\end{array}$ & $\begin{array}{c}\text { V. Basílica }(22 \%) \text {; V. } \\
\text { Cefálica }(20 \%) ; \text { V. Axilar } \\
(13,3 \%)\end{array}$ \\
\hline $\begin{array}{l}\text { FRANCESCH; } \\
\text { CUNHA (2010) }\end{array}$ & - & Neonatologia & $\begin{array}{l}\text { Oclusão (19,44\%); Ruptura } \\
\text { (8,8\%); Infiltração }(5,09 \%) ; \\
\text { Sepse Clínica }(4,16 \%)\end{array}$ & - \\
\hline
\end{tabular}

Legenda: AVE = Acidente Vascular Encefálico; CA = Câncer; DPOC = Doença Pulmonar Obstrutiva Crônica; DRP = Desconforto Respiratório Precoce; ICC = Insuficiência Cardíaca Congestiva; MMII = Membros Inferiores; MMSS = Membros Superiores; SDR = Síndrome do Desconforto Respiratório; V = veia, $\mathrm{UTI}=$ unidade de terapia intensiva.

\section{Discussão}

\section{Perfil predominante do paciente em uso do PICC, principais diagnósticos e especialida- des médicas que fazem uso do cateter}

Os pacientes submetidos ao PICC são, na maioria, $\mathrm{RN}$ prematuros e de baixo peso, que necessitam do cateter para garantir que a terapêutica medicamentosa seja implementada e, assim, tenham maiores chances de sobrevida e melhor prognóstico ${ }^{9}$, visto que apresentam risco aumentado de mortalidade, sendo a prematuridade responsável por cerca de um terço dos óbitos neonatais. No Brasil, a taxa de mortalidade nesse grupo é de 7,9 a $14,2 \% 5$.

$\mathrm{Na}$ prematuridade, a reserva metabólica do RN é baixa e o mesmo exige aporte exógeno para o desempenho de seus processos fisiológicos, isso através da infusão de hidratação com eletrólitos, carboidratos, proteínas e lipídeos ${ }^{11}$. No caso de RN de baixo peso são maiores as chances de utilizar o PICC por estarem mais debilitados, com maior risco de infecção, por terem sua termorregulação e perfusão prejudicadas, e porque os órgáos relacionados à sucçáo e nutriçáo ainda não estáo plenamente desenvolvidos, além de apresentarem outras complicaçóes ${ }^{12-13}$.

A maior susceptibilidade a distúrbios respiratórios são resultado da imaturidade anatômica e fisiológica dos prematuros, que são propensos também à retinopatia, hemorragias peri e intraventriculares, restrição do crescimento, dentre outras ${ }^{5,14}$.

A predominância de $\mathrm{RN}$ do sexo masculino está relacionada às complicaçóes da prematuridade (síndrome do desconforto respiratório ou doença da membrana hialina), que tem como fatores de risco a própria prematuridade (neonatos com peso entre 501 e 1.500 gramas), sexo masculino e asfixia perinatal ${ }^{15}$.

A utilização predominante do PICC na neonatologia pode ter relação com a característica clinica do paciente, a dificuldade de manutenção por um tempo prolongado do cateter venoso periférico e da pouca ou nenhuma utilizaçáo de cateteres tipo Hickman, Broviac e Port-a-cath ${ }^{\circ}$.

\section{Condutas associadas, tempo médio de per- manência e locais de inserção}

A inserção do cateter deve ser indicada precocemente para que haja sucesso na utilização e no RN assim que ele tenha condiçóes de ser submetido ao procedimento, pois punçôes venosas repetidas, distorçôes anatômicas, cirúrgicas ou traumáticas geram vasos sanguíneos muito delgados, de difícil identificação, comprometidos por dor, edema, eritema e hematomas, o que dificulta a inserção e manutenção do cateter. $\mathrm{O}$ procedimento é eletivo e não deve ser realizado na urgência e emergência, devido aos riscos que apresenta ${ }^{12,16-18}$.

O PICC pode permanecer por tempo indeterminado desde náo haja complicaçóes e o cuidado apropriado seja realizado ${ }^{6}$, porém o CDC recomenda a utilização por 8 semanas $^{5}$, e há estudos que indicam o uso por até 6 meses $^{12,15,19-20}$. Quanto maior o tempo de permanência, maior o risco de infecção $o^{21}$, no entanto, cateteres posicionados centralmente estão associados a baixas taxas de complicação (tromboflebite, flebite e oclusão) ${ }^{13}$ comparados aos cateteres não centrais ${ }^{22}$.

$\mathrm{Na}$ escolha da veia para inserção do PICC, deve ser observado se ela é palpável, calibrosa e com menos curvatura. A pele no local escolhido deve estar íntegra, sem hematomas, edemas, sinais de infecção ou alterações anatômicas ${ }^{1-2,20}$. 
As veias mais indicadas são a basílica e a cefálica, pois apresentam menor número de válvulas, maior calibre, têm anatomia favorável, facilitam a realizaçáo e troca de curativos e têm menor trajeto até a veia cava superior ${ }^{5-6,9,12,15,18,23}$, no entanto a veia cefálica, por ser menor que a basílica e possuir mais válvulas, oferece risco potencial para flebite e mal posicionamento ${ }^{1}$.

A escolha do membro superior direito oferece maior facilidade de progressão e centralização do cateter $^{24}$, além de estar mais próximo da veia cava ${ }^{25}$. As áreas de articulação devem ser evitadas, priorizando-se as veias mais distais e superficiais ${ }^{15}$.

As veias localizadas na cabeça, como a temporal, auricular posterior e jugular externa apresentam maior risco de migração do cateter, portanto devem ser a última opção, e as veias dos MMII como a poplítea, safena e femoral possuem válvulas que podem dificultar a progressão do cateter ${ }^{23}$. Embora a veia jugular externa seja a mais visível, ela oferece maior risco de infecção pelo local. Na punção dela, recomenda-se optar pela direita, pois a esquerda pode ascender para a jugular interna ${ }^{20}$. A veia auricular é frágil e de tamanho variável, e assim como a jugular, tem difícil fixaçáo, a jugular externa e axilar oferecem risco de punção acidental da artéria carótida, com consequente hematoma, pneumotórax ou embolia gasosa. No entanto, a axilar permite uso de cateter de maior calibre e maior número de lúmens, devido a seu maior diâmetro ${ }^{25}$.

Nos prematuros, a artéria poplítea é mais visível, porém deve ser puncionada com cautela. A veia safena, pelo excesso de válvulas (7 a 15), apresenta maior dificuldade técnica e pode favorecer o desenvolvimento de edemas de MMII. A veia femoral é de difícil punção por localizar-se abaixo do ligamento inguinal ${ }^{2}$ e apresenta maior risco de formação de trombos e maior incidência de infecção relacionada ao cateter ${ }^{26}$.

O calibre do PICC deve ser definido pelo profissional, levando-se em consideração o ajuste do diâmetro do lúmen para o peso, idade do paciente e tamanho do vaso ${ }^{15}$.

Para mensurar o comprimento do cateter a ser inserido, o RN é colocado em decúbito dorsal, com o braço a ser puncionado num ângulo de $90^{\circ}$ em relação ao tronco e a cabeça voltada para o lado ipsilateral do membro que será cateterizado ${ }^{27}$. Para punçáo em MMSS, cabeça e pescoço, mede-se a distância entre o local de punção até a clavícula direita e o terceiro espaço intercostal e para MMII a distância da punção até região inguinal, passando por região umbilical até o apêndice xifoide ${ }^{17,20}$.

$\mathrm{Na}$ implantação do PICC deve ser adotada técnica de barreira máxima e feita a monitorizaçâo cardiorrespiratória, além de sedação e analgesia para prevenção da dor ${ }^{4}$, visto que a exposição a múltiplos procedimentos dolorosos e estresse geram maior gasto metabólico e esgotamento das reservas energéticas, desfavorecendo o ganho de peso, retardando a recuperação e/ou agravando as condiçốes clínicas dos $\mathrm{RN}^{14}$. São medidas analgésicas: a contençãa, sucção não nutritiva com sacarose ou leite humano, distração com músicas suaves, inclusão dos pais no procedimento, aplicação de mistura eutética de prilocaína e lidocaína a 5\% (EMLA) e analgesia medicamentosa ${ }^{9,27}$ com fentanil endovenoso (EV), midazolan $\mathrm{EV}$ ou via nasal ou hidrato de cloral via oral ${ }^{26}$. Em $\mathrm{RN}$, não se utiliza fio-guia na inserção, especialmente em cateteres siliconados, devido ao risco de fratura do dispositivo ${ }^{17}$.

O tempo de inserção é de cerca de 45 minutos $^{6,20}$ a uma hora ${ }^{16}$. Deve ser realizada de forma lenta, de 0,5 a $0,5 \mathrm{~cm}$, prevenindo, assim, a flebite mecânica ${ }^{17}$. Em média é inserido de 10 a $15 \mathrm{~cm}$ do cateter, medida que depende da veia e membro escolhido ${ }^{13}$.

O CDC de 2002 (categoria IA) indica para antissepsia cutânea no ato de inserção do cateter o gluconato de clorexedina aquosa $2 \%$, polivinil-pirrolidona iodo a $10 \%$, álcool a $70 \%$, ou clorexidina alcoólica a $0,5 \%{ }^{17}$. No entanto, o uso do antisséptico depende do peso do RN, em funçáo da fragilidade da pele. Para RN com peso inferior a $1.500 \mathrm{~g}$, é recomendado clorexidina degermante e com peso igual ou maior a $1.500 \mathrm{~g}$, clorexidina alcoólica, e em ambos, solução fisiológica (SF) 0,9\% para remoçáo do antisséptico". Já a clorexidina está indicada para menores de duas semanas por poder desencadear reação cutânea ${ }^{26}$.

$\mathrm{Na}$ radiografia para confirmação da localização da ponta do cateter pode ser utilizada as vértebras torácicas e a anatomia venosa do tórax como referenciais anatômicos. Quando a ponta do PICC estiver alojada entre a $3^{a}$ e $4^{a}$ vértebra torácica, está em veia cava superior. Ao nível do diafragma ou próximo da $9^{a}$ e $10^{a}$ vértebra torácica, em veia cava inferior ${ }^{25}$. Pode também ser utilizada a ultrassonografia ${ }^{26}$.

A dificuldade na inserção do cateter pode ocorrer devido a sangramento excessivo, obstrução por válvulas venosas e anatomia venosa aberrante. $\mathrm{O}$ dispositivo pode seguir outras ramificaçôes venosas e não ser posicionado centralmente, sendo necessário proceder com manobras de reposicionamento dos membros, tração do cateter e nova tentativa de progressão, além de instilação de SF para estimular a abertura das válvulas venosas e progressão do $\mathrm{PICC}^{25}$. Outras causas de dificuldade de progressão são: resistência no trajeto causado por venoespasmo, esclerose, posiçáo incorreta do cateter ou do paciente, bifurcação venosa ${ }^{12}$ e dissecção de veia prévia ${ }^{1}$.

A ponta do cateter mal posicionada pode trazer complicaçóes como arritmia cardíaca ${ }^{13}$, derrames pleurais e pericárdicos, tamponamento cardíaco, perfuração do miocárdio, trombose vascular, bacteremia, endocardite, 
sepse, embolia pulmonar após fratura, migração do cateter ${ }^{6}$, hemorragia, hematoma, punçáo arterial, dano e estimulação de nervos ${ }^{1}$, fístula arteriovenosa ${ }^{28}$, migraçáo do cateter para o cérebro, pneumonite ${ }^{11}$, perfuração e extravasamento em pelve renal e até morte ${ }^{12}$.

O curativo no local de inserção do cateter tem finalidade de cobrir, prevenir trauma local e contaminação ${ }^{29}$, proteger a inserção do cateter e evitar seu deslocamento ${ }^{1-2,4,20}$ é um procedimento estéril e exclusivo do enfermeiro habilitado. Na presença de drenagem de líquidos ou sangue no sítio de inserçâo é recomendado o curativo com gaze e fita adesiva, que deve ser trocado entre 48 a 72 horas, ou antes, se a integridade estiver comprometi$\mathrm{da}$, se o local estiver limpo e seco utiliza-se a membrana de poliuretano semipermeável, que possibilita melhor visualização e permanência prolongada, por até 7 dias $^{29}$. Há estudos que indicam o curativo transparente, que é impermeável e permite a evaporação de água, logo após a inserção, sendo feita a troca após 24 horas $^{1,20}$ e trocas subsequentes a cada 7 dias $^{18,30}$.

\section{Conclusão}

O PICC é uma tecnologia que se encontra em expansão dentro do ambiente hospitalar. Sobrepóe-se em vantagens quando comparado aos CVC e traz a possibilidade de acompanhamento ambulatorial, o que é positivo na qualidade de vida do paciente e na sua recuperação. Ainda permite a inserção pelo enfermeiro habilitado, no entanto, a falta de conhecimentos tecnocientíficos e preparo da equipe fazem que as taxas de complicaçóes ainda sejam relevantes, o tempo de permanência seja muito menor que o recomendado, além de ser uma lacuna para a disseminaçáo da prática em outras especialidades médicas além da neonatologia.

Evidencia-se que o enfermeiro tem grande importância nesse processo, tanto como agente de prevenção de complicaçóes, como disseminador da prática, mas o que se nota são poucas publicaçóes referentes ao assunto. Denota-se a relevância de se incluir a temática na formaçáo acadêmica, assim como o investimento do profissional em pesquisas nas mais diversas áreas, viabilizando assim resultados cada vez mais positivos na implantação dessa técnica.

\section{Referências}

1. Freitas EM, Nunes ZB. O enfermeiro na práxis de cateter central de inserção periférica em neonato. Rev. Min. Enferm. 2009;13(2):215-24.

2. Vieira AO, Campos FMC, Almeida DR, Romão DF, Aguilar VD, Garcia EC. Cuidados de enfermagem em pacientes neonatos com cateter central de inserção periférica. Rev. Gest Saúde. 2013;4(2):188-99.

3. Silva Bretas TC, Fagundes S, Ferreira M, Versiani CDC, Marques Andrade F. Conhecimento da equipe de enfermagem sobre inserção e manutençáo do cateter central de inserção periférica em recém nascidos. Enferm Global. 2013(32):21.

4. Belo MPM, Silva RAMC, Nogueira ILM, Mizoguti DP, Ventura CMU. Conhecimento de enfermeiros de neonatologia acerca do Cateter Venoso Central de Inserção Periférica. Rev bras enferm. 2012;65(1):42-8.

5. Rosa IC, Oselame GB, Oliveira EM, Almeida Dutra D, Neves EB. Caracterizaçáo do uso do cateter central de inserção periférica em uma UTI Neonatal no Estado do Paraná. RUVRD. 2014;12(1):536-46.

6. Montes S, Teixeira J, Barbosa M, Barichello E. Ocorrência de complicaçóes relacionadas ao uso de cateter venoso central de inserção periférica (PICC) em recém-nascidos. Enferm Global. 2011;24(4):10-8.

7. Costa LC, Paes GO. Aplicabilidade dos diagnósticos de enfermagem como subsídios para indicação do cateter central de inserção periférica. Esc Anna Nery Rev Enferm. 2012;16(4):649-56.

8. Conselho Federal de Enfermagem. Resolução no 258, de 12 de julho de 2001. Inserção de cateter periférico central pelos enfermeiros, 2001.

9. Dórea E, Castro TE, Costa P, Kimura AF, Santos FG. Práticas de manejo do cateter central de inserção periférica em uma unidade neonatal. Rev Bras Enferm. 2011;64(6):997-1002.

10. Galvão C, Sawada N, Trevizan M. Revisão sistemática. Rev Latino-Am Enfermagem. 2004;12(3):549-56.

11. Reis T, Santos S, Barreto J, Silva G. O uso do cateter epicutâneo na clientela neonatal de um hospital público estadual: estudo retrospectivo. Rev Enferm UERJ. 2011;19(4):592-7.

12. Neves MP, Fialho FA, Dias IMÁV, Nascimento L. Cateter central de inserção periférica: o papel da enfermagem na sua utilização em neonatologia. HU Revista. 2012;37(2):163-68.

13. Camargo P, Kimura AF, Toma E, Tsunechiro MA. Localização inicial da ponta de cateter central de inserção periférica (PICC) em recém-nascidos. Rev Esc Enferm USP. 2008;42(4):723-8.

14. .Costa P, Camargo PP, Bueno M, Kimura AF. Dimensionamento da dor durante a instalação do cateter central de inserção periférica em neonatos. Acta Paul Enferm. 2010;23(1):35-40.

15. Baggio MA, Bazzi FCS, Bilibio CAC. Cateter central de inserção periférica: descrição da utilização em UTI Neonatal e Pediátrica. Rev Gaúcha de Enferm. 2010;31(1):70-6.

16. Vendramim P, Pedreira MLG, Peterlini MAS. Cateteres centrais de inserção periférica em crianças de hospitais do município de São Paulo. Rev Gaúcha de Enferm. 2007;28(3):331. 
17. Lourenço A, Ohara C. Conhecimento dos enfermeiros sobre a técnica de inserção do cateter central de inserção periférica em recém-nascidos. Rev Latino-Am Enfermagem. 2010;18(2):189-95.

18. Rodrigues ZS, Chaves EMC, Cardoso M. Atuação do enfermeiro no cuidado com o cateter central de inserçáo periférica no recém-nascido. Revista Bras Enferm. 2006;59(5):626-9.

19. Lamblet LCR, Guastelli LR, Júnior DFM, Aparecida M, Alves Y, Bittencourt AC, et al. Cateter central de inserção periférica em terapia intensiva de adultos. Rev Bras Ter Intensiva. 2005;17(1):23-7.

20. Nunes SAS, Oliveira LN. Atuação do enfermeiro na inserção, manutenção e remoção do Cateter Central de Inserção Periférica. Rev Enferm UNISA. 2007;8:67-71.

21. Duarte ED, Pimenta AM, Noelly e Silva BC, Paula CM. Fatores associados à infecçáo pelo uso do cateter central de inserção periférica em Unidade de Terapia Intensiva Neonatal. Rev Esc Enferm USP. 2013;47(3):547-54.

22. Baiocco GG, Silva J. A utilização do cateter central de inserção periférica (CCIP) no ambiente hospitalar. Rev LatinoAm Enfermagem. 2010;18(6):1131-7.

23. Gomes A, Nascimento M, Antunes J, Araújo M. El cateter percutáneo en la unidad de cuidados intensivos neonatales: una tecnología del cuidado de enfermería. Enferm Global. 2010;19:1-13.
24. Swerts CAS, Felipe AOB, Miranda Rocha K, Andrade CUB. Cuidados de enfermagem frente às complicaçôes do cateter central de inserção periférica em neonatos. Revista Eletrônica de Enfermagem. 2013;15(1):156-61.

25. Costa P, Vizzotto MPS, Olivia CL, Kimura A. Sítio de inserção e posicionamento da ponta do cateter epicutâneo em neonatos. Rev Enferm UERJ. 2013;21(4):452-7.

26. Johann DA, Lazzari LSMD, Pedrolo E, Mingorance P, Almeida TQR, Danski MTR. Peripherally inserted central catheter care in neonates: an integrative literature review. Rev Esc de Enferm USP. 2012;46(6):1503-11.

27. Costa P, Bueno M, Oliva CL, Castro TE, Camargo PP, Kimura AF. Analgesia e sedaçáo durante a instalacao do cateter central de inserção periférica em neonatos. Rev Esc Enferm USP. 2013;47(4):801-7.

28. Franceschi AT, Cunha MLC. Eventos adversos relacionados ao uso de cateteres venosos centrais em recém-nascidos hospitalizados. Rev Latino-Am Enfermagem. 2010;18(2):196-202.

29. Johann DA, Danski MTR, Pedrolo E, Lazzari LSM, Mingorance P. Avaliação de um cuidado de enfermagem: o curativo de catéter central de inserção periférica no recém-nascido. REME. 2010;14(4):515-20.

30. Coelho NA, Namba M. Ceteter Central de Inserção Periférica: cuidados de enfermagem em neonatologia. Rev Enferm UNISA 2009;10(2):167-71.

\section{Como citar este artigo:}

Martins C, Oselame GB, Neves EB. Cateter central de inserção periférica: revisão sistemática. Rev. Aten. Saúde. 2016;14(47): 99-107. 